\title{
Surface plasmon LED with graphene-GaN grating structure HuiboWang ${ }^{1, ~ a ~}$, ZhibinWang ${ }^{2, b}$ \\ ${ }^{1}$ School of Continuing Education, Yanshan University, Qinhuangdao-066004, China \\ ${ }^{2}$ School of Electric Engineering, Yanshan University, Qinhuangdao-066004, China \\ awanghuibo@ysu.edu.cn
}

\section{Keywords:Optical Devices; Light Emitting Diodes; Light Extraction Efficiency; Surface PlasmonPolar tons}

Abstract.In order to increase light extraction efficiency of GaN-based light emitting diodes (LED), surface plasmon (SP) LED, with a graphene grating structure is proposed and modelled. In this structure, the traditional silver film is replaced by a grapheme layer to produce SP. The surface plasmon enhancement factor of the transmitted light is analyzed theoretically to explain the influence of this structure on the improvement of LED light extraction efficiency. The finite difference time domain (FDTD) method is used for numerical simulation, showing that the light extraction efficiency of LEDs with graphene grating structure is increased by about 1.7 times than LEDs with the silver film structure. The conclusion provides theoretical support for the design and optimization of GaN-based LED with high performance.

\section{Introduction}

In recent years, with the development of nanotechnology, the use of nanostructures to generate SP (Surface Plasmon) and improve the LED (Light Emitting Diodes) luminous efficiency has become a hot research topic ${ }^{[1]}$. Okmoto et al.placed a silver film on top of the active layer of a LED device with the InGaN quantum well structure, and has achieved 14 times increase on photoluminescence intensity, along with the internal quantum efficiency 6.8 times improvement ${ }^{[2]}$. C.Huh et al. added a layer of silver nanoparticles in silicon-based LED ${ }^{[3]}$.

They have achieved silicon nanocrystal photoluminescence intensity two orders of magnitude improvement and the light extraction efficiency of the device is enhanced by 50\%[4]. Most researchers use silver to excite SP[5,6]. However, for the light within the escape cone, Ag will become an obstacle to external radiation and there is a great loss when SP propagate on the silver film surfaces. Those factors limit the further improvement of luminous efficiency.

To solve the problem and to improve light extraction efficiency of LED devices, in this work, a new design of LED structure is proposed. This LED structure uses graphene to replace the silver film and adds a grating on surface to increase the SP radiation. Our results show that SP can enhance the transmission of light. The light emitting efficiency of this graphene-GaN grating LED is compared with the graphene-containing and the Ag-containing LED structures. Our results demonstrate that the LED with graphene-GaN grating can further improve the LED luminous efficiency.

\section{Theories}

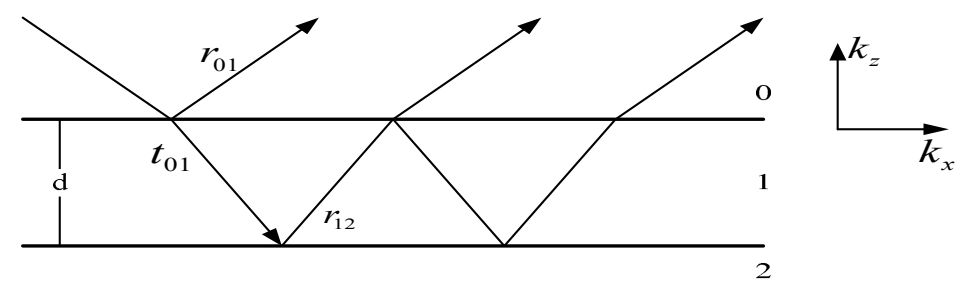

Fig.1 Schematic of light propagation in a metal film

Surface plasmon in the form of $\mathrm{H}$-wave (magnetic field parallel to the surface) propagate along the metal surface, and in the direction of perpendicular to the surface (dielectric or metal) exponentially fast decay. When the thickness of the metal is limited, surface plasmon can be 
generated on the surface of both sides of the metal plate [7], coupled by penetrating the metal. Ultimately the surface plasmon of symmetric and antisymmetric with a certain frequency vibration is formed on both surfaces of the metal plate [8]. The electromagnetic field strength of surface plasmon with symmetric and antisymmetric on the surface of both sides of the metal plate are the same, thus they will result in extraordinary optical penetration[9,10].

As shown in Figure 1, the light incident into the metal film from the GaN (0/1 interface), part of the light is reflected and the other part of the light is transmitted into the metal film. Part of the light transmitted into the metal filmis reflected at 1/2 interface, and producesfurther reflection and transmission at $0 / 1$ interface. ${ }^{E_{1 / 2}}$ represents the electric field strength to the $1 / 2$ interface, and ${ }^{E_{0 / 1}}$ represents the electric field strength to the $0 / 1$ interface. The enhancement factor of single interface can be obtained by Maxwell equations and Fresnel formulas as:

$$
t_{i k}=1+\left(\frac{k_{z i}}{\varepsilon_{i}}-\frac{k_{z k}}{\varepsilon_{k}}\right) /\left(\frac{k_{z i}}{\varepsilon_{i}}+\frac{k_{z k}}{\varepsilon_{k}}\right)
$$

Where $k_{z i}=\sqrt{\varepsilon_{i}\left(\frac{\omega}{c}\right)^{2}-\left(k_{x}^{0}+\Delta k_{x}\right)^{2}}, i, k$ is $0 、 1,2$.

$$
k_{x}^{0}=\frac{\omega}{c} \sqrt{\frac{\varepsilon_{0}}{\varepsilon_{0}+1}} \Delta k_{x}=k_{x}-\sqrt{\left(\frac{\omega}{c}\right)^{2}-k_{z}^{2}}
$$

So the total enhancement factor of the light passes through the metal film is

$$
\left|t_{012}\right|^{2}=\frac{\left|E_{1 / 2}\right|^{2}}{\left|E_{0 / 1}\right|^{2}}=\frac{\varepsilon_{0}}{\varepsilon_{1}} \frac{t_{01} t_{12} e^{i \alpha}}{1+r_{01} r_{12} e^{2 i \alpha}}
$$

With $\mathrm{r}_{i k}=t_{i k}-1, \alpha=k_{z 1} d$,

$$
t_{012}=2 t_{01} \frac{w}{c} e^{i \alpha}\left(\frac{\varepsilon_{1} \varepsilon_{2}}{\varepsilon_{1}+\varepsilon_{2}}\right)^{3 / 2} \frac{\varepsilon_{0}}{\varepsilon_{2}\left(\varepsilon_{1}-\varepsilon_{2}\right)} \frac{1}{k_{x}-\left(k_{x}^{0}+\Delta k_{x}\right)}
$$

When $\lambda=450 \mathrm{~nm}, \varepsilon_{2}=1$ (air), $\varepsilon_{0}=2.2$, assuming metal film is Ag film, ${ }^{t_{012}}$ about 100 . Thus, the SP are very important for radiate light in high-refractive index material.

Graphene is a single crystal functional material, and has a two-dimensional honeycomb lattice structure which is a single layer of carbon atoms tightly packed together [11]. Graphene has unique electrical and optical properties [12,13] . Graphene can support SP better than precious metals[14,15], partly because there are more favorable mode diffusion (defined as $1 / \operatorname{Re}(\beta)$ ) and transmission length (defined as $1 / \operatorname{Im}(\beta)$ ) of SP in graphene; Furthermore, the conductivity properties of graphene can be adjusted by the applied bias or chemical doping.

\section{Device fabrication and simulation analysis}

\section{Device fabrication}

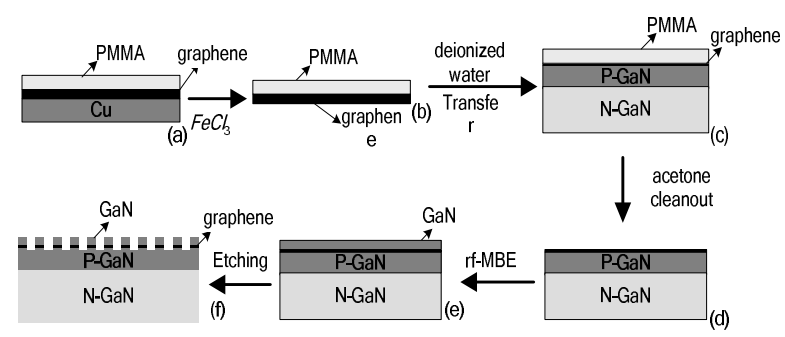

Fig.2 Preparation flowchart of LED of graphene grating structure 
The process flow for fabricating LEDs with a graphene grating structure is shown in figure 2 and the each process step is explained as follows:

1) Graphene is grown on a $\mathrm{Cu}$ substrate. PMMA is then coated on grapheme layer.

2) The PMMA grapheme / $\mathrm{Cu}$ structure was immersed in an $\mathrm{FeCl}$ aqueous solution, to etch away the $\mathrm{Cu}$ substrate.

3) The The PMMA/grapheme layers are washed in deionized water and transferred to a GaN-based LED surface.

4) The sample is soaked in acetone to remove PMMA.

5) $\mathrm{GaN}$ is deposited on the graphene surface in the manner of rf-MBE (radio-frequency molecular beam epitaxy).

6) Etch grating on the model surface.

\section{Simulation comparison}

A numerical simulation was conducted using the FDTD method. The refractive index of P-GaN layer and N-GaN layer is 2.4. The graphene dielectric coefficient is the complex dielectric constant, obtained by formula (11). The silver film was used in the Lorentz dispersion model. Complex permittivity and complex permeability was used to indicative the loss of light in silver film. In the GaN LED structure, a single electric dipole source was used to simulate quantum well light emitting, and the wavelength of dipole source is $550 \mathrm{~nm}$.

To illustrate the enhancement of graphene grating LED to light extraction efficiency, the other three models are constructed as a comparison group: silver film LED with a silver film is deposited on P-GaN; graphene LED with graphene is placed on P-GaN; Ag grating LED with Ag layer is inserted in $\mathrm{GaN}$ and grating is etched. These structures are analyzed by simulation, compareing the influence of graphene layer and the grating on the light extraction efficiency, respectively.

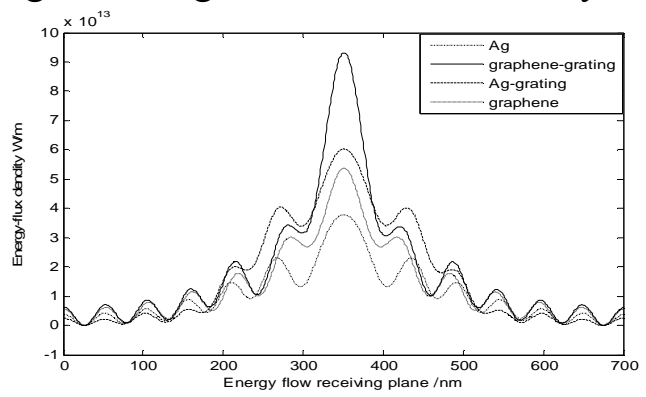

Fig.3 Energy flow of each model comparison chart

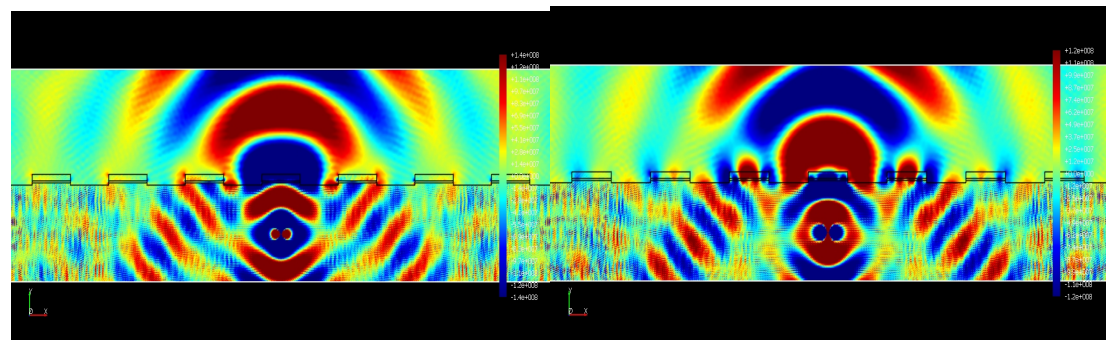

(a) graphene grating structure

(b) Ag grating structure

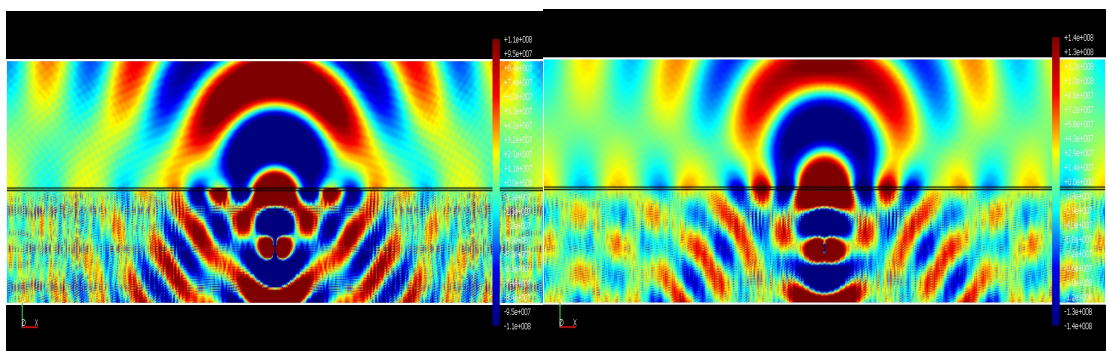

(c) graphene structure

(d) Ag film structure

Fig.4 Real- time filed of LED model comparison chart 
In Figure 3, energy flow of each model comparison chart, contrast the curve of Ag film structure and graphene structures can be seen, mostly the outgoing light concentrated in $300 \mathrm{~nm}-400 \mathrm{~nm}$ of receiving plane, this part is above the light source, and the light emission is within the escape cone. Due to the light absorption and reflection, the silver film at this condition turned out to hinder light radiating. Light transmittance of graphene is up to $97 \%$. Therefore, at the $300 \mathrm{~nm}-400 \mathrm{~nm}$, energy flow of the LED with a graphene structure was significantly higher than the silver film structure. Contrast the curve of grating structures and non-grating structures can be seen, the energy flow of grating structures is high than non-grating structures on entire receiving plane. This is mainly affected by two factors, first, through grating, SP convert into visible light; Second, grating damages the total reflection occurred $\mathrm{GaN}$ and air interface. As can be seen from Figure 4, without grating, SP is bound in silver film and the graphene surface, unable to radiate outwards, gradually spread on surface, and become heat loss. Though GaN grating, SP convert into visible light, and the light emits to the entire receiving plane.

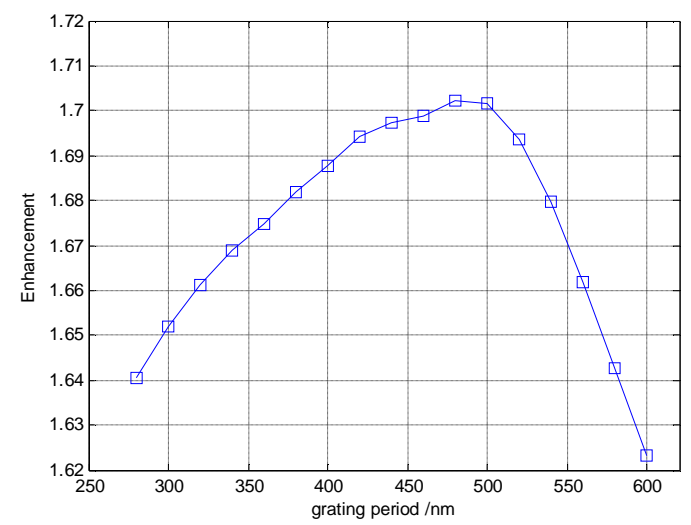

Fig.5the enhancement of grapheme grating structure relative to the $\mathrm{Ag}$ film structure at different grating periods As can be seen from Figure 5, when the grating has a period of 480nm, the light extraction efficiency of the LED with a graphene grating structure is the highest, energy flow increased by about 1.7 times than the LED with a Ag film. This result is attributed to two factors. First, the surface grating period is related to the wave vector that causes plasmon resonance. Thus, only when the grating period satisfies the excitation conditions can the SP be excited. Consequently, visible light is extracted and transmissible light is enhanced. Second, the ideal size of diffraction grating satisfies the wavelength of light on a metal surface when the period is considerably smaller than the wavelength of light. Otherwise, conductivity to light diffraction is low and the effect of changing light directions caused by light diffraction decreases. On the other hand, when the period is far from the wavelength of light, the plane of the grating section is excessively large, thereby causing stronger total internal reflection, which slows down light emission.

\section{Conclusion}

The LED model with graphene grating structure based on surface plasma is designed, Maxwell equations and Fresnel formulas are used for analysis, and SP enhancement factor of transmission light is derived. The process can be achieved by using realizable and precise production methods. The FDTD method is introduced for the numerical simulation, with results showing that the light extraction efficiency of LED with graphene grating structure is higher than LED with the Ag film structure. The value is increased by about 1.7 times.

\section{References:}

[1] J M pitarke,V M Silkin, E V Chulkov,et al."Theory of surface plasmons and surface-plasmons polaritons". Rep. Prog.Phys.2007,70(1):01-03. 
[2] K.Okamoto, I.Niki, A.Shvartser, Y.Narukawa, T. Mukai, A. Scherer. "Surface-plasmon-enhanced light emitters based on InGaN quantum wells. Nature Mater" . 2004, 3(9): 601-605.

[3] C. Huh, C.-J. Choi,W. Kim, B. K. Kim, B.-J. Park, E.-H. Jang,S,H. Kim, G. Y. Sung.Enhancement in light emission efficiency of Si nanociystal light-emitting diodes by a surface plasmon coupling. Appl. Phys. Lett.,2012,100(18): 181108.

[4] C. Tong, J. Yun,H. Song, Q. Gan, W. A. Anderson. "plasmonic-enhanced Si Schottky barrier solar cells”. Sol. Energy Mater. Sol. Cells, 2014, 120(B); 591-595.

[5]X.-Y. Wang, J.-L. Wang, H. Wang. "Improvement of the efficiency and power output of solar cells using nanoparticles and annealing”. Solar Energy, 2014, 101: 100-104.

[6]S. K. Sardana, V. S. N. Chava, E. Thouti, N. Chander, S. Kumar, S. R. Reddy, V. K.Komarala. "Influence of surface plasmon resonances of silver nanoparticles on optical and electrical properties of textured silicon solar cell”. AppL Phys. Lett, 2014, 104(7): 073903.

[7] E. Matioli and C. Weisbuch. "impact of photonic crystals on LED light extraction emciency: approaches and limits to vertical structure designs", J. Phys. D:Phys.2010 43, 354005-354005-22.

[8]M. Ohtsu, K. Kobayashi, T. Kawazoe et al. "Nanophotonics: design, fabrication, and operation of nanometric devices using optical near fields", Selected Topics in Quantum Electronics, IEEE Journal of vol. 2002, 8:839 862

[9] Y. Z. Lin,K. Li,F. M. Kong,et al. "Comprehensive Numeric Study of Gallium Nitride Light-emitting Diodes Adopting Surface plasmon Mediated Light Emission Technique" .IEEE Journal of Selected Topics in Quantum Electronics, 2011, 17(4):942-951.

[10] H. Raether, "Surface plasmons on Smooth and Rough Surface and on Gratings", Springer, Berlin, Germany, 1998.

[11] Han P Y, Liu W, Xie Y H, Zhang X C, “Graphene and terahertz science”,Physics,2009,38(6), $395-400$

[12]Geim A K, MacDonald A H, “Graphene: Exploring carbon flatland”, Phys. Today, 2007, 60(8), $35-41$

[13]Castro Neto A H, Guinea F, Peres N M R, Novoselov K S, Geim A K. "The electronic properties of graphene", Rev. Mod. Phys, 2009, 81(109),1-55

[14]Ashkan Vakil, Nader Engheta, "Transformation Optics Using Graphene”, Science, 2011, 332(6035),1291-1294

[15]Ashka n Vakil, Nader Engheta. "Transformation Optics Using Graphene” .Science, 2011, 332(10): 1291 1294 